\title{
Standard and quasi-conformal BFKL kernels
}

\author{
V.S. Fadin ${ }^{* \dagger}$ \\ Budker Institute of Nuclear Physics of SD RAS, 630090 Novosibirsk Russia \\ and \\ Novosibirsk State University, 630090 Novosibirsk, Russia \\ E-mail: fadin@inp.nsk.su
}

\begin{abstract}
The ambiguity of the NLO BFKL kernels in consequence of the possibility to rearrange radiative corrections to scattering amplitudes between the impact factors and the Green functions permits to write the kernels for the singlet and adjoint representations of the gauge group in the quasiconformal appearance, i.e. in the form where conformal invariance is violated only by running coupling. The differences between standard and quasi-conformal kernels in the momentum space are found.
\end{abstract}

XXI International Workshop on Deep-Inelastic Scattering and Related Subject-DIS2013, 22-26 April 2013

Marseilles,France

\footnotetext{
* Speaker.

† Work supported in part by the Ministry of Education and Science of Russian Federation and in part by RFBR, grant 13-02-01023.
} 


\section{Introduction}

The BFKL (Balitsky-Fadin-Kuraev-Lipatov) approach [1] based on the remarkable property of non-Abelian gauge theories — reggeization of gauge bosons (gluons) — is applicable for all possible representations of the gauge (colour) group in the $t$ - channel. Now the kernel of the BFKL equation is known in the next-to-leading order (NLO) both for forward scattering, i.e. for $t=0$ and the colour singlet in the $t$-channel [2], and for arbitrary $t$ and any possible colour state in the $t$ - channel [3].

For phenomenological applications, the most interesting is the Pomeron (colour singlet) channel. But the adjoint representation is not less important. For two reggeized gluons the adjoint representation can be of two different types, symmetric and antisymmetric ones. In fact, antisymmetric adjoint representation is even more important, than the singlet one, first of all because of the gluon reggeization. The idea of the gluon reggeization appeared as the result of the fixed order calculations. Evidently it must be proved. It can be done [4] using bootstrap relations, which follow from the requirement of compatibility of the multi-Regge form of amplitudes with the $s$-channel unitarity. Now fulfillment of these relations is proved in the NLO (see [5] and references therein).

But there are at least two other reasons for significance of the adjoint representations. One is related to the BKP (Bartels-Kwiecinski-Praszalowicz) equation [6] - the generalization of the BFKL equation to bound states consisting of three and more reggeized gluons, in particular the C-odd three gluon system - Odderon. The BFKL kernel for symmetric adjoint representation appears in the BKP equation for the odderon because any pair of the three reggeized gluons are in the colour octet state. Lately, the kernel of the BKP equation was found in the NLO [7].

Recently, another application of the BFKL approach, connected with the BDS (Bern-DixonSmirnov) ansatz [8] for amplitudes with maximal helicity violation in Yang-Mills theories with extended supersymmetry ( $N=4 \mathrm{SYM}$ ) at large number of colours $N_{c}$ was extensively developed [9]. The approach was used for verification of the BDS ansatz for inelastic amplitudes and calculation of the remainder factor. It was demonstrated that the BDS amplitude $M_{2 \rightarrow 4}^{B D S}$ should be multiplied by the factor containing the contribution of the Mandelstam cuts, and this contribution was found in the LLA [9] and in the NLA [10]. Note that at large $N_{c}$, when only planar diagrams are taken into account, there is the signature degeneracy, i.e. there is no difference between symmetric ant antisymmetric adjoint representations. In the following talking about the adjoint representation we will will assume just this case.

In the leading order (LO) the kernels of the BFKL equation for the singlet and the adjoint representations of the gauge group have the remarkable property which is named by the same word conformal invariance, although its meaning is different for different representations: in the first case it means invariance with respect to the Möbius transformations in the space of transverse coordinates, whereas in the second case - in the space of transverse momenta. In the NLO the conformal invariance is violated by running coupling. It is natural to think that the NLO kernels are quasiconformal, i.e. their conformal invariance is violated only by terms proportional to the $\beta$ function. But the kernels defined according to [11] (we call them standard) are not quasi-conformal. However, it does not mean that there are another origins of violation of conformal invariance besides the running coupling. The reason is that NLO kernels are scheme-dependent because of the possibility to rearrange radiative corrections to scattering amplitudes in the BFKL approach between 
the impact factors and the kernels.

For the singlet kernel, it was shown [12] that this ambiguity permits to write the kernel in the quasi-conformal appearance. It turned out that in the impact parameter space the quasi-conformal kernel is extremely simple compared with the standard one. is i.e. in the form where conformal invariance is violated only by running coupling. In the next Section we discuss the difference between two forms of this kernel.

For the kernel in the adjoint representation, it was believed, but not proved, that at $N=4$ SYM at large $N_{c}$ it can be written in a conformal invariant form. In the Section 3 we demonstrate that indeed the standard kernel can be reduced to the conformal invariant one by the similarity transformation.

\section{Colour singlet kernel}

For scattering of colourless objects, due to gauge invariance of the impact factors, the BFKL equation can be written in the Möbius invariant form [13]. In this form the BFKL kernel acts in the space of functions turning into zero at coinciding transverse coordinates. The Möbius invariance of the LO BFKL kernel can be made evident by transformation from the transverse momentum to the transverse coordinate representation [14].

The Möbius form obtained by transformation of the standard NLO kernel [11] into impact parameter space turned out not quasi-conformal[15]. But the ambiguity of NLO kernels, analogous to the well known ambiguity of the NLO anomalous dimensions, permits to make transformations

$$
\hat{K} \rightarrow \hat{K}-\alpha_{s}\left[\hat{K}^{(B)}, \hat{U}\right] .
$$

Using this transformation it turns out possible to reduce the Möbius form to the quasi-conformal shape [12], which is immeasurably simple compared with the standard kernel in the momentum space.

Especially simple it is at $N=4 \mathrm{SYM}$ :

$$
\begin{gathered}
\left\langle\vec{r}_{1} \vec{r}_{2}\left|\hat{\mathscr{K}}_{M}^{C}\right| \vec{r}_{1}^{\prime} \vec{r}_{2}^{\prime}\right\rangle_{N=4}=\frac{\alpha_{s} N_{c}}{2 \pi^{2}} \int d \vec{\rho} \frac{\vec{r}_{12^{2}}^{2}}{\vec{r}_{1 \rho}^{2} \vec{r}_{2 \rho}^{2}} \\
\times\left[\delta\left(\vec{r}_{11^{\prime}}\right) \boldsymbol{\delta}\left(\vec{r}_{2^{\prime} \rho}^{2}\right)+\delta\left(\vec{r}_{1^{\prime} \rho}\right) \boldsymbol{\delta}\left(\vec{r}_{22^{\prime}}\right)-\delta\left(\vec{r}_{11^{\prime}}\right) \boldsymbol{\delta}\left(r_{22^{\prime}}\right)\right]\left(1-\frac{\alpha_{s} N_{c} \zeta(2)}{2 \pi}\right) \\
+\frac{\alpha_{s}^{2} N_{c}^{2}}{4 \pi^{4}}\left[\frac{\ln \left(\frac{\vec{r}_{1 \prime^{\prime}}^{2} \vec{r}_{1^{\prime}}^{2}}{\vec{r}_{11^{\prime}}^{2} \vec{r}_{22^{\prime}}^{2}}\right)}{2 \vec{r}_{11^{\prime}}^{2} \vec{r}_{22^{\prime}}^{2}}\left(\frac{\vec{r}_{12}^{4}}{\vec{r}_{12^{\prime}}^{2} \vec{r}_{21^{\prime}}^{2}-\vec{r}_{11^{\prime}}^{2} \vec{r}_{22^{\prime}}^{2}}-\frac{\vec{r}_{12}^{2}}{\vec{r}_{1^{\prime} 2^{\prime}}^{2}}\right)+\frac{\vec{r}_{12}^{2} \ln \left(\frac{\vec{r}_{12}^{2} \vec{r}_{12^{\prime}}^{2}}{\vec{r}_{11^{\prime}}^{2} \vec{r}_{21^{\prime}}^{2}}\right)}{\vec{r}_{11^{\prime}}^{2} \vec{r}_{22^{\prime}}^{2} \vec{r}_{1^{\prime} 2^{\prime}}^{2}}\right. \\
\left.+6 \pi^{2} \zeta(3) \delta\left(\vec{r}_{11^{\prime}}\right) \delta\left(r_{22^{\prime}}\right)\right],
\end{gathered}
$$

In fact, there are three reasons for the simplicity:

- Möbius representation (i.e. limitation of space of states),

- transformation $\hat{K} \rightarrow \hat{K}-\alpha_{s}\left[\hat{K}^{B}, \hat{U}\right]$,

— use of impact parameter space. 
The simplicity of the Möbius form of the quasi-conformal NLO BFKL kernel suggested to use just this form for finding the kernel in the momentum space. The way to do that was not evident, and even the possibility to do it seemed doubtful, because the Möbius form is defined on a special class of functions in the coordinate space. However, it was shown [16] that such possibility exists due to the gauge invariance of the kernel, and the way to obtain the kernel in the momentum space from its Möbius form was elaborated. But technically obtaining it turned out to be not easy. Instead, the difference between the standard and the quasi-conformal BFKL kernels was found [17].

An explicit form of the operator $\hat{U}$ in the momentum space [16]

$$
\left\langle\vec{q}_{1}, \vec{q}_{2}\left|\alpha_{s} \hat{U}\right| \vec{q}_{1}^{\prime}, \vec{q}_{2}^{\prime}\right\rangle=\delta\left(\vec{q}_{11^{\prime}}+\vec{q}_{22^{\prime}}\right) \frac{\alpha_{s} N_{c}}{4 \pi^{2}} R_{u}\left(\vec{q}_{1}, \vec{q}_{2} ; \vec{k}\right)-\frac{\alpha_{s} \beta_{0}}{8 \pi} \ln \left(\vec{q}_{1}^{2} \vec{q}_{2}^{2}\right) \delta\left(\vec{q}_{11^{\prime}}\right) \delta\left(\vec{q}_{22^{\prime}}\right),
$$

where $\beta_{0}$ is the first coefficient of the Gell-Mann-Low function,

$$
\beta_{0}=\frac{11}{3} N_{c}-\frac{2}{3} n_{f}
$$

and

$$
\begin{gathered}
R_{u}\left(\vec{q}_{1}, \vec{q}_{2} ; \vec{k}\right)=\frac{1}{\vec{q}_{1}^{2}} \ln \left(\frac{\vec{q}_{1}^{\prime 2} \vec{q}_{2}^{2}}{\vec{k}^{2} \vec{q}^{2}}\right)+\frac{1}{\vec{q}_{2}^{2}} \ln \left(\frac{\vec{q}_{2}^{\prime 2} \vec{q}_{1}^{2}}{\vec{k}^{2} \vec{q}^{2}}\right)+\frac{1}{\vec{k}^{2}} \ln \left(\frac{\vec{q}_{1}^{\prime 2} \vec{q}_{2}^{\prime 2}}{\vec{q}_{1}^{2} \vec{q}_{2}^{2}}\right) \\
-2 \frac{\vec{q}_{1} \vec{k}}{\vec{k}^{2} \vec{q}_{1}^{2}} \ln \left(\frac{\vec{q}_{1}^{\prime 2}}{\vec{k}^{2}}\right)+2 \frac{\vec{q}_{2} \vec{k}}{\vec{k}^{2} \vec{q}_{2}^{2}} \ln \left(\frac{\vec{q}_{2}^{\prime 2}}{\vec{k}^{2}}\right)-2 \frac{\vec{q}_{1} \vec{q}_{2}}{\vec{q}_{1}^{2} \vec{q}_{2}^{2}} \ln \left(\frac{\vec{q}^{2}}{\vec{k}^{2}}\right) .
\end{gathered}
$$

Note that $R_{u}$ has the gauge invariance properties:

$$
\begin{gathered}
R_{u}\left(\vec{q}_{1}, \vec{q}_{2} ; \vec{q}_{1}\right)=R_{u}\left(\vec{q}_{1}, \vec{q}_{2} ;-\vec{q}_{2}\right)=0, \\
\left.\left(\vec{q}_{1}^{2} \vec{q}_{2}^{2} R_{u}\left(\vec{q}_{1}, \vec{q}_{2} ; \vec{k}\right)\right)\right|_{\vec{q}_{1}=0}=\left.\left(\vec{q}_{1}^{2} \vec{q}_{2}^{2} R_{u}\left(\vec{q}_{1}, \vec{q}_{2} ; \vec{k}\right)\right)\right|_{\vec{q}_{2}=0}=0 .
\end{gathered}
$$

Indeed, these properties are required to conserve the gauge invariance.

The difference between the standard and the quasi-conformal kernels turned out to be rather simple

$$
\begin{gathered}
D\left(\vec{q}_{1}, \vec{q}_{1}^{\prime} ; \vec{q}\right)=\frac{\alpha_{s}^{2} N_{c}^{2}}{8 \pi^{3}}\left[-\frac{\beta_{0}}{2 N_{c}}\left(\frac{2}{\vec{k}^{2}}-2 \frac{\vec{q}_{1} \vec{k}}{\vec{k}^{2} \vec{q}_{1}^{2}}+2 \frac{\vec{q}_{2} \vec{k}}{\vec{k}^{2} \vec{q}_{2}^{2}}-2 \frac{\vec{q}_{1} \vec{q}_{2}}{\vec{q}_{1}^{2} \vec{q}_{2}^{2}}\right) \ln \left(\frac{\vec{q}_{1}^{\prime 2} \vec{q}_{2}^{\prime 2}}{\vec{q}_{1}^{2} \vec{q}_{2}^{2}}\right)\right. \\
+\frac{\vec{q}_{1}^{\prime 2}}{\vec{q}_{1}^{2} \vec{k}^{2}} \ln \left(\frac{\vec{q}_{1}^{2} \vec{q}_{2}^{\prime 2}}{\vec{q}_{2}^{2} \vec{q}_{1}^{\prime 2}}\right) \ln \left(\frac{\vec{q}_{2}^{2} \vec{q}_{1}^{\prime 2}}{\vec{q}^{2} \vec{k}^{2}}\right)+\frac{\vec{q}_{2}^{\prime 2}}{\vec{q}_{2}^{2} \vec{k}^{2}} \ln \left(\frac{\vec{q}_{2}^{2} \vec{q}_{1}^{\prime 2}}{\vec{q}_{1}^{2} \vec{q}_{2}^{\prime 2}}\right) \ln \left(\frac{\vec{q}_{1}^{2} \vec{q}_{2}^{\prime 2}}{\vec{q}^{2} \vec{k}^{2}}\right) \\
-4\left(\frac{\left[\vec{q}_{1} \times \vec{q}_{2}\right]}{\vec{q}_{1}^{2} \vec{q}_{2}^{2}}+\frac{\left[\vec{q}_{1} \times \vec{k}\right]}{\vec{q}_{1}^{2} \vec{k}^{2}}+\frac{\left[\vec{q}_{2} \times \vec{k}\right]}{\vec{q}_{2}^{2} \vec{k}^{2}}\right)\left(\left[\vec{q}_{1} \times \vec{q}_{2}\right] I_{\vec{q}_{1}, \vec{q}_{2}}-\left[\vec{q}_{1}^{\prime} \times \vec{q}_{2}^{\prime}\right] I_{\left.\left.\vec{q}_{1}^{\prime}, \vec{q}_{2}^{\prime}\right)\right]}\right.
\end{gathered}
$$

where

$$
I_{\vec{p}, \vec{q}}=\int_{0}^{1} \frac{d x}{(\vec{p}+x \vec{q})^{2}} \ln \left(\frac{\vec{p}^{2}}{x^{2} \vec{q}^{2}}\right) .
$$

The most natural conclusion is that the simplicity of the Möbius form of the quasi-conformal kernel is caused mainly by using the impact parameter space. The other possibility is that the quasiconformal kernel can be written in simple form also in the transverse momentum space. If this is true, the standard kernel could result itself in a much simpler form. 


\section{Adjoint representation kernel}

For calculation of high-energy asymptotic of the remainder function, the modified (with subtracted gluon trajectory depending on total $t$-channel momenta) BFKL kernel in the adjoint representation is used. Assuming that this kernel can be written in the form, which is invariant with respect to the Möbius transformations in the momentum space, the eigenfunctions and eigenvalues of the kernel were found in [10]. The conformal invariant kernel is completely determined by these eigenfunctions and eigenvalues. It takes the form [18]:

$$
K_{c}\left(\vec{q}_{1}, \vec{q}_{1}^{\prime} ; \vec{q}\right)=K^{B}(z)\left(1-\frac{\alpha_{s} N_{c}}{2 \pi} \zeta(2)\right)+\delta^{(2)}(1-z) \frac{\alpha_{s}^{2} N_{c}^{2}}{4 \pi^{2}} 3 \zeta(3)+\frac{\alpha_{s}^{2} N_{c}^{2}}{32 \pi^{3}} R(z),
$$

where

$$
\begin{aligned}
K^{B}(z) & =\frac{\alpha_{s} N_{c}}{8 \pi^{2}}\left(\frac{z+z^{*}}{|1-z|^{2}}-\delta^{(2)}(1-z) \int \frac{d \vec{l}}{|l|^{2}} \frac{l+l^{*}}{|1-l|^{2}}\right), \\
R(z)= & \left(\frac{1}{2}-\frac{1+|z|^{2}}{|1-z|^{2}}\right) \ln ^{2}|z|^{2}-\frac{1-|z|^{2}}{2|1-z|^{2}} \ln |z|^{2} \ln \frac{|1-z|^{4}}{|z|^{2} \mid} \\
+ & \left(\frac{1}{1-z}-\frac{1}{1-z^{*}}\right)\left(z-z^{*}\right) \int_{0}^{1} \frac{d x}{|x-z|^{2}} \ln \frac{|z|^{2}}{x^{2}},
\end{aligned}
$$

$z=q_{1} q_{1}^{\prime} / q_{1}^{\prime} q_{2}, p=p_{x}+i p_{y}$ and $p^{*}=p_{x}-i p_{y}$ for any two-dimensional vector $\vec{p}=\left(p_{x}, i p_{y}\right)$. If we denote

$$
K\left(\vec{q}_{1}, \vec{q}_{1}^{\prime} ; \vec{q}\right)-K_{c}\left(\vec{q}_{1}, \vec{q}_{1}^{\prime} ; \vec{q}\right)=\frac{\alpha_{s}^{2} N_{c}^{2}}{32 \pi^{3}} \Delta\left(\vec{q}_{1}, \vec{q}_{1}^{\prime} ; \vec{q}\right)
$$

then

$$
\begin{aligned}
& \Delta\left(\vec{q}_{1}, \vec{q}_{1}^{\prime} ; \vec{q}\right)=\ln \frac{\vec{q}_{1}^{2}}{\vec{q}^{2}} \ln \frac{\vec{q}_{2}^{2}}{\vec{q}^{2}}+\ln \frac{\vec{q}_{1}^{\prime 2}}{\vec{q}^{2}} \ln \frac{\vec{q}_{2}^{\prime 2}}{\vec{q}^{2}}+\ln \frac{\vec{q}_{1}^{2}}{\vec{q}_{1}^{\prime 2}} \ln \frac{\vec{q}_{2}^{2}}{\vec{q}_{2}^{\prime 2}} \\
& -2 \frac{\vec{q}_{1}^{2} \vec{q}_{2}^{\prime 2}+\vec{q}_{2}^{2} \vec{q}_{1}^{\prime 2}}{\vec{k}^{2} \vec{q}^{2}} \ln \frac{\vec{q}_{1}^{2}}{\vec{q}_{1}^{\prime 2}} \ln \frac{\vec{q}_{2}^{2}}{\vec{q}_{2}^{\prime 2}}+\frac{\vec{q}_{1}^{2} \vec{q}_{2}^{\prime 2}-\vec{q}_{2}^{2} \vec{q}_{1}^{\prime 2}}{\vec{k}^{2} \vec{q}^{2}}\left(\ln \frac{\vec{q}_{1}^{2}}{\vec{q}^{2}} \ln \frac{\vec{q}_{2}^{\prime 2}}{\vec{q}^{2}}-\ln \frac{\vec{q}_{2}^{2}}{\vec{q}^{2}} \ln \frac{\vec{q}_{1}^{\prime 2}}{\vec{q}^{2}}\right) \\
& +\frac{4}{\vec{q}^{2} \vec{k}^{2}}\left(\vec{k}^{2}\left[\vec{q}_{1} \times \vec{q}_{2}\right]-\vec{q}_{1}^{2}\left[\vec{k} \times \vec{q}_{2}\right]-\vec{q}_{2}^{2}\left[\vec{k} \times \vec{q}_{1}\right]\right)\left(\left[\vec{q}_{1} \times \vec{q}_{2}\right] I_{\vec{q}_{1}, \vec{q}_{2}}-\left[\vec{q}_{1}^{\prime} \times \vec{q}_{2}^{\prime}\right] I_{\vec{q}_{1}^{\prime}, \vec{q}_{2}^{\prime}}\right),
\end{aligned}
$$

where $\vec{k}=q_{1}-\vec{q}_{1}^{\prime}=\vec{q}_{2}^{\prime}-\vec{q}_{2}$. Important properties of $\Delta$ are its symmetries with respect to the exchanges $\vec{q}_{1} \leftrightarrow-\vec{q}_{2}, \vec{q}_{1}^{\prime} \leftrightarrow-\vec{q}_{2}^{\prime}$ and $\vec{q}_{i} \leftrightarrow-\vec{q}_{i}$, as well as the gauge invariance (vanishing at zero momentum of each reggeon), which are easily seen from this representation.

If the kernels $\hat{K}$ and $\hat{K}_{c}$ coincide in the leading order and are connected by a similarity transformation, there must exist an operator $\hat{O}$ satisfying the commutation relation

$$
\left[\hat{K}^{B}, \hat{O}\right]=\left(\frac{\alpha_{s}}{2 \pi}\right)^{2} \frac{1}{8 \pi} \hat{\Delta} .
$$

It was shown [18] that indeed such operator exists and its explicit form was found:

$$
\hat{\mathscr{O}}_{t}=\frac{1}{4}\left[\ln \left(\hat{\vec{q}}_{1}^{2} \hat{\vec{q}}_{2}^{2}\right), \hat{\mathscr{K}}_{r}^{B}\right]=\frac{\alpha_{s} N_{c}}{16 \pi^{2}}\left(\frac{\vec{q}_{1}^{2} \vec{q}_{2}^{\prime 2}+\vec{q}_{1}^{\prime 2} \vec{q}_{2}^{2}}{\vec{k}^{2}}-\vec{q}^{2}\right) \ln \left(\frac{\vec{q}_{1}^{2} \vec{q}_{2}^{2}}{\vec{q}_{1}^{\prime 2} \vec{q}_{2}^{\prime 2}}\right) .
$$

It means that indeed the modified BFKL kernel in the adjoint representation of the gauge group at $N=4 \mathrm{SYM}$ can be written in the conformal invariant form. 


\section{References}

[1] V.S. Fadin, E.A. Kuraev and L.N. Lipatov, Phys. Lett. B60 (1975) 50;

E.A. Kuraev, L.N. Lipatov and V.S. Fadin, Sov. Phys. JETP 44 (1976) 443, ibid. 45 (1977) 199;

I.I. Balitsky and L.N. Lipatov, Sov. J. Nucl. Phys. 28 (1978) 822.

[2] V.S. Fadin and L.N. Lipatov, Phys. Lett. B429 (1998) 127;

M. Ciafaloni and G. Camici, Phys. Lett. B430 (1998) 349;

A. V. Kotikov and L. N. Lipatov, Nucl. Phys. B 582 (2000) 19.

[3] V. S. Fadin, R. Fiore and A. Papa, Phys. Rev. D 60 (1999) 074025;

V. S. Fadin and D. A. Gorbachev, JETP Letters 71 (2000) 222; Phys. Atom. Nucl. 63 (2000) 2157;

V. S. Fadin and R. Fiore, Phys. Rev. D72 (2005) 014018; Phys. Lett. B 661 (2008) 139;

R. E. Gerasimov, V. S. Fadin, Phys. Atom. Nucl. 73 (2010) 1214.

[4] Ya. Ya. Balitskii, L. N. Lipatov and V. S. Fadin, in Materials of IV Winter School of LNPI

(Leningrad, 1979) p.109;

V. S. Fadin, R. Fiore, M. G. Kozlov and A. V. Reznichenko, Phys. Lett. B 639 (2006) 74.

[5] M. G. Kozlov, A. V. Reznichenko and V. S. Fadin, Phys. Atom. Nucl. 75 (2012) 493.

[6] J. Bartels, Nucl. Phys. B 175 (1980) 365;

J. Kwiecinski and M. Praszalowicz, Phys. Lett. B 94 (1980) 413.

[7] J. Bartels, V. S. Fadin, L. N. Lipatov and G. P. Vacca, arXiv:1210.0797 [hep-ph].

[8] Z. Bern, L. J. Dixon and V. A. Smirnov, Phys. Rev. D 72 (2005) 085001.

[9] J. Bartels, L. N. Lipatov and A. Sabio Vera, Phys. Rev. D 80 (2009) 045002, Eur. Phys. J. C65 (2010) 587 ;

L. N. Lipatov and A. Prygarin, Phys. Rev. D 83 (2011) 045020,

Phys. Rev. D 83 (2011) 125001;

J. Bartels, L. N. Lipatov and A. Prygarin, Phys. Lett. B 705 (2011) 507.

[10] V. S. Fadin and L. N. Lipatov, Phys. Lett. B 706 (2012) 470.

[11] V.S. Fadin and R. Fiore, Phys. Lett. B440 (1998) 359. [arXiv:hep-ph/9807472].

[12] V. S. Fadin, R. Fiore and A. V. Grabovsky, Nucl. Phys. B831, 248 (2010).

[13] L.N. Lipatov, Sov. Phys. JETP 63, 904 (1986).

[14] V. S. Fadin, R. Fiore, and A. Papa, Nucl. Phys. B769, 108 (2007). Phys. Lett. B647, 179 (2007).

[15] V. S. Fadin, R. Fiore, A. V. Grabovsky, and A. Papa, Nucl. Phys. B784, 49 (2007).

[16] V.S. Fadin, R. Fiore, A.V. Grabovsky and A. Papa, Nucl. Phys. B856 (2012) 111.

[17] V. S. Fadin, R. Fiore and A. Papa, Nucl. Phys. B 865 (2012) 67 [arXiv:1206.5596 [hep-th]].

[18] V. S. Fadin, R. Fiore, L. N. Lipatov and A. Papa, arXiv:1305.3395 [hep-th]. 